\title{
CONCEITOS DE SAÚDE E DOENÇA: ANÁLISE DAS TENDÊNCIAS EM TESES E DISSERTAÇÕES BRASILEIRAS
}

\author{
Tatiana Pereira das Neves Gamarra
}

GAMARRA, T. P. da N. Conceitos de saúde e doença: análise das tendências em teses e dissertações brasileiras. Arq. Cienc. Saúde UNIPAR, Umuarama, v. 23, n. 1, p. 49-55, jan./abr. 2019.

\begin{abstract}
RESUMO: Refletir criticamente acerca dos conceitos de saúde e doença é extremamente relevante, uma vez que a formação e a prática dos profissionais de saúde que abrange historicidade, modificações e limites dos saberes dominantes estão permeadas por estas concepções. Nessa perspectiva, este artigo de revisão narrativa da literatura teve por objetivo analisar as tendências das teses e dissertações brasileiras acerca dos conceitos de saúde e doença. A busca foi realizada na Biblioteca Digital Brasileira de Teses e Dissertações. Para a análise utilizou-se a análise de conteúdo temática. Todos os estudos presentes na Biblioteca que abordaram os conceitos de saúde e doença foram examinados. Não houve recorte temporal. Foram incluídas as teses e dissertações que apresentaram a expressão "conceitos de saúde e doença" em quaisquer dos campos de busca disponíveis. 18 estudos foram identificados e os principais temas existentes nestas produções acadêmicas foram: perspectiva biomédica, perspectiva social e perspectiva subjetiva. Concluiu-se que os temas identificados não se excluem, ao contrário, são complementares, apontando as variadas possibilidades de compreensão dos conceitos citados. Todas as produções analisadas abordaram os três temas, porém, isto não significou o mesmo grau de destaque nos estudos. A perspectiva biomédica foi reconhecida como a mais consolidada nas práticas em saúde, embora os estudos indiquem a relevância das perspectivas social e subjetiva para uma compreensão ampliada do processo saúde-doença e também ressaltem dificuldades para a superação da perspectiva biomédica na atenção em saúde. PALAVRAS-CHAVE: Saúde. Doença. Atenção à Saúde.
\end{abstract}

\section{HEALTH AND DISEASE CONCEPTS: TREND ANALYSIS IN BRAZILIAN THESES AND DISSERTATIONS}

\begin{abstract}
Critical reflection on the concepts of health and disease is extremely relevant since the training and practice of health professionals encompassing historicity, modifications and limits of the dominant knowledge are permeated by such conceptions. In this perspective, this narrative literature review paper aims at analyzing the trends in the Brazilian theses and dissertations regarding the concepts of health and disease. In order to do so, a search was performed at the Brazilian Digital Library of Theses and Dissertations, using a thematic content analysis. All the studies in the Library that addressed the concepts of health and disease were examined. There was no temporal cut. Theses and dissertations presenting the expression "health and disease concepts" in any of the available search fields were included. A total of 18 studies were identified and the main themes in these academic papers were: biomedical perspective, social perspective, and subjective perspective. It could be concluded that the identified themes are not mutually excluding, on the contrary, they are complementary, pointing out the many possibilities of understanding these concepts. All the productions analyzed addressed the three topics. However, that does not mean that they had the same level of prominence in the studies. The biomedical perspective was recognized as the most consolidated among the health practices, although the studies indicate the relevance of the social and subjective perspectives for an expanded understanding of the health-disease process, also emphasizing the difficulties in overcoming the biomedical perspective in health care.
\end{abstract}

KEY WORDS: Health. Disease. Health Care.

\section{Introdução}

Refletir criticamente acerca dos conceitos de saúde e doença é extremamente relevante uma vez que a formação e a prática dos profissionais de saúde que abrange historicidade, modificações e limites dos saberes dominantes estão permeadas por tais concepções. É um tema que mobiliza os pesquisadores do campo da Saúde Coletiva, com discussões, análises e propostas inovadoras dos saberes e modos de intervir na realidade que não são novos e permanecem como desafios (CANESQUI, 2016).

Geralmente, a questão da saúde é analisada a partir de múltiplos saberes, com contribuições tanto das ciências humanas como das ciências médicas e biológicas com ideias nem sempre convergentes sobre o assunto. De uma perspectiva qualitativa, a saúde privilegia a subjetividade que é definida no interior de uma relação entre corpo e ambiente e se opõe ao modelo biomédico clássico, que busca usar a abordagem quantitativa como método nas definições de saúde.
Historicamente, na formação médica, considerou-se doença como desvio das normas previamente fixadas, estabelecidas por meio de estudos experimentais de laboratório. Assim, reestabelecer a saúde seria buscar um retorno do organismo a tais normas definidas de forma científica (RODRIGUES; PEIXOTO JÚNIOR, 2014).

Saúde e doença são conceitos críticos que possuem implicações sociais e políticas de longo alcance. Por exemplo, qualquer tentativa de formação dos profissionais ou regulação de planos de saúde deve utilizar alguns padrões para avaliar se as pessoas estão doentes ou não. Os conceitos de saúde e doenças também se conectam de forma interessante com questões sobre suas funções e explicações na filosofia das ciências biomédicas e teorias do bem-estar na ética (MURPHY, 2015).

Além disso, saúde e doença, como muitos outros conceitos, não são exclusivamente científicos nem de senso comum. Ao contrário apresentam uma base científica e também estão presentes no pensamento cotidiano. Isso suscita

DOI: 10.25110/arqsaude.v23i1.2019.6588

'Biomédica (UNIRIO), especialista em Saúde Pública (ENSP-FIOCRUZ), especialista em gestão pública em serviços de saúde (Faculdade Internacional Signorelli), especialista em auditoria em serviços de saúde (Faculdade Internacional Signorelli), especialista em gestão pública (Faculdade Internacional Signorelli), especialista em docência e pesquisa para a área de saúde (ISEAT), mestre em Saúde Pública (ENSP-FIOCRUZ) e doutora em Saúde Pública (ENSP-FIOCRUZ). Atua como especialista em regulação na Agência Nacional de Saúde Suplementar. E-mail: tatibiom@yahoo.com.br 
um problema para qualquer discussão filosófica: quando se tenta dizer o que a saúde e a doença realmente significam de modo estritamente científico, mas, ao mesmo tempo se busca distanciar-se do senso comum, com o que se deve preocupar? (MURPHY, 2015).

A filosofia contemporânea da saúde tem discutido bastante o problema de determinar a natureza dos conceitos de saúde e doença do ponto de vista científico. Alguns teóricos afirmam e argumentam que esses conceitos são isentos de valor e descritivos no mesmo sentido em que os conceitos de átomo, metal e chuva. Dessa forma, dizer que uma pessoa tem uma certa doença ou que é saudável é, portanto, descrever objetivamente essa pessoa. Por outro lado, tal descrição certamente não impede uma avaliação subjetiva do estado de cada pessoa. A descrição científica básica e a avaliação são duas questões independentes, de acordo com esse tipo de teoria (NORDENFELT, 2007).

Ainda segundo Nordenfelt (2007), outros filósofos, porém, afirmam que o conceito de saúde, como outros conceitos médicos, está essencialmente carregado de valor. Dessa maneira, estabelecer que uma pessoa é saudável não implica apenas uma inspeção e medição objetiva, mas, pressupõe também uma avaliação do estado geral da pessoa. Afirmar que alguém é saudável não implica somente alguns fatos científicos em relação ao corpo ou mente da pessoa, mas requer também uma avaliação (positiva) de seu estado corporal e mental .

Diante de tantas implicações e discussões é importante conhecer a produção acadêmica de teses e dissertações brasileiras sobre os conceitos de saúde e doença para saber o panorama ampliado sobre o tema, uma vez que tais estudos são provenientes de diferentes programas de pós-graduação, tendo, desse modo, olhares diversos sobre o assunto o que pode contribuir de forma relevante para a compreensão das implicações do tema.

Para orientar este estudo, formulou-se a seguinte questão de revisão: Quais as tendências das teses e dissertações brasileiras acerca dos conceitos de saúde e doença? Sendo assim, este estudo tem como objetivo analisar as tendências das teses e dissertações brasileiras acerca dos conceitos de saúde e doença".

\section{Metodologia}

Trata-se de um estudo de revisão da literatura, com abordagem narrativa. A revisão narrativa é caracterizada como uma pesquisa bibliográfica ampla, sendo adequada para descrever e analisar o desenvolvimento de um determinado assunto, sob o ponto de vista teórico ou contextual (ROTHER, 2007)

A busca das teses e dissertações foi realizada na Biblioteca Digital Brasileira de Teses e Dissertações (BDTD). Esta integra e divulga, em um único portal de busca, os textos completos das teses e dissertações defendidas nas instituições brasileiras de ensino e pesquisa, contribuindo para o aumento de conteúdos de teses e dissertações brasileiras na internet, proporcionando maior visibilidade à produção científica nacional e favorecendo a difusão de informações de interesse científico e tecnológico para a sociedade (BRASIL, 2017). Foram analisadas todas as teses e dissertações presentes na mencionada biblioteca que continham os conceitos de saúde e doença em quaisquer campos de busca disponíveis. Espera-se dessa maneira identificar os principais temas existentes nessas pesquisas, bem como discutir semelhanças e diferenças de compreensão em relação aos citados conceitos. Para a seleção das teses e dissertações não foi estabelecido recorte temporal, pois, pretendeu-se realizar uma análise abrangente do tema.

Utilizou-se para a análise dos dados a análise de conteúdo temática, conforme Bardin (2011). Segundo a autora, essa técnica tem como função principal o revelar crítico de mensagens de maneira que se possa inferir sobre outra realidade que não aquela presente na mensagem. A análise de conteúdo temática define-se como conjunto de estratégias de caráter metodológico em constante aperfeiçoamento, aplicado a conteúdos extremamente variados. Suas características fundamentais são o foco em mensagens (comunicações) e a natureza categorial-temática e se divide em três etapas: a pré-análise, a exploração do material e o tratamento dos resultados".

\section{Resultados}

A busca foi realizada em novembro de 2017, utilizando-se como palavra-chave a expressão "conceitos de saúde e doença”. A pesquisa resultou em 23 estudos encontrados.

Desse total, foram excluídas duas produções acadêmicas porque estavam repetidas, três estudos também foram descartados por não contemplar a discussão dos conceitos de saúde e doença. Assim, foram examinadas 18 produções acadêmicas, sendo 12 dissertações e 06 teses.

Após leitura detalhada de cada uma das produções acadêmicas encontradas, foi possível identificar três grandes temas que emergiram destes estudos: perspectiva biomédica, perspectiva social e perspectiva subjetiva. Tais temas não se excluem e podem, inclusive, apresentar uma sobreposição, já que algumas vezes um mesmo estudo pode abordar mais de um tema, contudo, são importantes como norteadores da análise, ajudando na compreensão da complexidade dos conceitos de saúde e doença.

Para auxiliar a sistematização da discussão foi elaborado um quadro-síntese dos estudos analisados- quadro 1, destacando seus principais objetivos e o tipo de estudo: 
Quadro 1: Síntese dos estudos analisados

\begin{tabular}{|c|c|c|}
\hline Título, autor, ano de publicação & Tipo & Objetivos \\
\hline $\begin{array}{l}\text { Conceitos afirmativos de saúde e doença: } \\
\text { um enlace entre os pensamentos de Georges } \\
\text { Canguilhem e Donald Winnicott, Rodrigues, } \\
2015\end{array}$ & Tese & $\begin{array}{l}\text { Analisar os conceitos de saúde e doença nas teorias de } \\
\text { Georges Canguilhem e Donald Winnicott na perspectiva de } \\
\text { que existem afinidades no pensamento destes autores }\end{array}$ \\
\hline $\begin{array}{l}\text { Fonoaudiologiaesaúdemental: considerações } \\
\text { sobre o trabalho fonoaudiológico em um } \\
\text { centro de atenção psicossocial, Carotta, } 1996\end{array}$ & Dissertação & $\begin{array}{l}\text { Analisar as perspectivas do trabalho fonoaudiológico num } \\
\text { centro de Atenção Psicossocial por meio de uma descrição } \\
\text { da prática fonoaudiológica nesta instituição }\end{array}$ \\
\hline $\begin{array}{l}\text { A química da qualidade de vida: um olhar } \\
\text { antropológico sobre uso de medicamentos e } \\
\text { saúde em classes médias urbanas brasileiras, } \\
\text { Azize, } 2002\end{array}$ & Dissertação & $\begin{array}{l}\text { Demonstrar que, dentro do sistema biomédico, diferentes } \\
\text { significados podem ser atribuídos às ideias de doença/saúde, } \\
\text { cura e medicamento; esta diferença fica dependente de quem } \\
\text { emite o discurso, do lugar social a partir do qual está falando, } \\
\text { com quem e com quais fins. }\end{array}$ \\
\hline $\begin{array}{l}\text { A Criança e sua rede familiar: significações } \\
\text { do processo de hospitalização, Menezes, } \\
2010\end{array}$ & Tese & $\begin{array}{l}\text { Compreender as significações do processo de hospitalização } \\
\text { a partir da perspectiva da criança hospitalizada e seu } \\
\text { acompanhante }\end{array}$ \\
\hline $\begin{array}{l}\text { A concepção de professores sobre saúde na } \\
\text { escola, Ferraro, } 2011\end{array}$ & Dissertação & $\begin{array}{l}\text { Conhecer qual a concepção sobre Saúde na Escola de } \\
\text { professores das escolas públicas estaduais inseridas no } \\
\text { Projeto Saúde e Prevenção nas Escolas da cidade de Franca }\end{array}$ \\
\hline $\begin{array}{l}\text { O corpo adoecido em seu seio: subjetividade } \\
\text { e câncer de mama, Dall'Olio, } 2006\end{array}$ & Dissertação & $\begin{array}{l}\text { Investigar as implicações subjetivas do câncer de mama e da } \\
\text { mastectomia a partir do estudo de um caso clínico realizado } \\
\text { em uma instituição hospitalar }\end{array}$ \\
\hline $\begin{array}{l}\text { A constituição do si-mesmo: uma abordagem } \\
\text { winnicottiana, Marchesini, } 2010\end{array}$ & Dissertação & $\begin{array}{l}\text { Conceituar e descrever o processo de constituição do } \\
\text { si-mesmo saudável à luz da teoria winnicottiana do } \\
\text { amadurecimento humano }\end{array}$ \\
\hline $\begin{array}{l}\text { O ensino de enfermagem em saúde mental e } \\
\text { psiquiátrica no Paraná, Maftum, } 2004\end{array}$ & Tese & $\begin{array}{l}\text { Conhecer como acontece o ensino de enfermagem em } \\
\text { saúde mental e psiquiátrica nos cursos de graduação em } \\
\text { enfermagem do Paraná }\end{array}$ \\
\hline $\begin{array}{l}\text { Estudo comparativo entre os conceitos de } \\
\text { saúde e de doença mental e a assistência } \\
\text { psiquiátrica, segundo portadores e familiares, } \\
\text { Osinaga, 2004 }\end{array}$ & Tese & $\begin{array}{l}\text { Comparar as opiniões dos portadores de doença mental e seus } \\
\text { familiares sobre conceitos de saúde e doença mental e sobre } \\
\text { a assistência em psiquiatria, em três serviços de assistência } \\
\text { em saúde mental. }\end{array}$ \\
\hline $\begin{array}{l}\text { O mito atividade física/saúde, Carvalho, } \\
1993\end{array}$ & Dissertação & $\begin{array}{l}\text { Investigar a relação que se estabeleceu e que se perpetua entre } \\
\text { a atividade física e a saúde, particularmente na sociedade } \\
\text { brasileira nas duas últimas décadas }\end{array}$ \\
\hline $\begin{array}{l}\text { Atenção à saúde de gestantes, puérperas e } \\
\text { bebês: o agir em competência de equipes de } \\
\text { saúde na atenção primária à saúde, Gomes, } \\
2014\end{array}$ & Dissertação & $\begin{array}{l}\text { Analisar o agir em competência na produção do cuidado às } \\
\text { gestantes, puérperas e bebês de Equipes de Saúde da Família } \\
\text { com Equipes de Saúde Bucal implantadas e que atuam em } \\
\text { Unidades Básicas de Saúde }\end{array}$ \\
\hline $\begin{array}{l}\text { A avaliação geriátrica ampla: a contribuição } \\
\text { da enfermagem na promoção da saúde do } \\
\text { idoso, Ribeiro, } 2011\end{array}$ & Dissertação & $\begin{array}{l}\text { Investigar a configuração da Avaliação Geriátrica Ampla } \\
\text { (AGA) e os efeitos da sua utilização no âmbito do Programa } \\
\text { Viva melhor a melhor idade e, também, para a discutir a } \\
\text { contribuição da enfermagem na promoção de saúde do idoso }\end{array}$ \\
\hline $\begin{array}{l}\text { Percepção de risco em relaçao à saúde bucal } \\
\text { de indivíduos cadastrados em um Núcleo } \\
\text { de Saúde da Família de Ribeirão Preto-SP, } \\
\text { Grigoletto, } 2007\end{array}$ & Dissertação & $\begin{array}{l}\text { Conhecer a percepção de risco, relacionada à saúde bucal, de } \\
\text { indivíduos cadastrados em um Núcleo de Saúde da Família } \\
\text { (NSF), no município de Ribeirão Preto-SP, por meio da } \\
\text { identificação das crenças, atitudes e conhecimentos desses } \\
\text { indivíduos sobre os fatores de risco relacionados ao tema }\end{array}$ \\
\hline $\begin{array}{l}\text { Prática Curativa: Um Saber Sonegado? } \\
\text { Pumar-Cantini, 2005 }\end{array}$ & Dissertação & $\begin{array}{l}\text { Refletir sobre questões relacionadas às crenças que } \\
\text { sustentam a manutenção de práticas populares de cura, de } \\
\text { como esse saber atravessa gerações sociológicas por meio da } \\
\text { cosmovisão de rezadeiras e receitadores }\end{array}$ \\
\hline $\begin{array}{l}\text { Perfil social das pessoas com hipertensão } \\
\text { arterial sistêmica atendidas no Programa de } \\
\text { Saúde da Família, Martins, } 2008\end{array}$ & Dissertação & $\begin{array}{l}\text { Conhecer, por amostragem, o perfil da população portadora } \\
\text { de hipertensão arterial sistêmica (HAS) atendida nas Equipes } \\
\text { de Saúde da Família (ESF). }\end{array}$ \\
\hline
\end{tabular}




\begin{tabular}{|l|l|l|}
\hline $\begin{array}{l}\text { Novo BAROS: análise crítica da metodologia } \\
\text { e sugestões para aprimoramento do BAROS, } \\
\text { Nicareta, 2010 }\end{array}$ & Tese & $\begin{array}{l}\text { Analisar criticamente a constituição e metodologia do } \\
\text { BAROS e sugerireimplementar mudanças neste questionário, } \\
\text { visando desenvolver novo instrumento de análise }\end{array}$ \\
\hline $\begin{array}{l}\text { Concepções de saúde e doença mental: } \\
\text { adaptação transcultural do instrumento } \\
\text { de acesso aos cuidados em saúde mental, } \\
\text { Morais, } 2014\end{array}$ & Tese & $\begin{array}{l}\text { Apresentar evidências de validade do instrumento Acesso } \\
\text { as Cuidados em Saúde Mental (ACSM), nome dado a } \\
\text { versão brasileira do instrumento Access to Mental Health } \\
\text { Care (AMHC) }\end{array}$ \\
\hline $\begin{array}{l}\text { Representações de saúde e doença por } \\
\text { profissionais da Saúde no contexto } \\
\text { pluricultural de fronteira, Duarte, 2014 }\end{array}$ & Dissertação & $\begin{array}{l}\text { Compreender aspectos que envolvem as diferenças culturais } \\
\text { e suas representações sociais: como os profissionais da } \\
\text { Saúde no cenário pluricultural de fronteira constroem, em } \\
\text { suas práticas discursivas, representações sobre saúde-doença }\end{array}$ \\
\hline
\end{tabular}

Elaboração própria

\section{Discussão}

\section{Perspectiva biomédica}

Diversos autores como Rodrigues (2015), Carotta (1996), Azize (2002), Menezes (2010), Ferraro (2011), Dall'Olio (2006), Marchesini (2010), Maftum (2004), Osinaga (2004), Carvalho (1993), Gomes (2014), Ribeiro (2011), Grigoletto (2007), Pumar-Cantini (2005), Martins (2008), Nicareta (2010), Morais (2014), Duarte (2014), abordam a perspectiva biomédica dos conceitos de saúde e doença, isto significa que todos os estudos examinados contemplam este tema.

Concepções de saúde e de doença tendem a ir além da simples condição biológica de algum estado. No caso da saúde, ainda existem perspectivas que consideram que um indivíduo saudável é apenas alguém cuja biologia funciona como algumas teorias apontam. Contudo, em relação ao conceito de doença, a maioria dos estudiosos que escrevem sobre o assunto adicionam outras condições ligadas à qualidade de vida (MURPHY, 2015).

Nesse sentido, todas as produções acadêmicas examinadas referem-se à necessidade de um olhar ampliado para a compreensão do processo saúde-doença que contemple aspectos biológicos, sociais, psicológicos, econômicos, dentre outros. A crítica à insuficiência da visão restrita biológica dos conceitos de saúde e doença está bastante presente em vários estudos analisados. Ribeiro (2011) em sua dissertação de mestrado, destaca que a metáfora corpo/máquina impôs uma visão fragmentada sobre o corpo e como consequência trouxe fragmentação ao cuidado em saúde.

Já Maftum (2004) em sua tese de doutorado ressalta que a hegemonia da perspectiva biomédica sobre a compreensão do processo saúde e doença produziu implicação na formação dos enfermeiros com os currículos de graduação, habilitando o profissional a tratar das necessidades físicas/ biológicas dos pacientes.

Em sua dissertação de mestrado, Duarte (2014) aponta que o modelo biomédico que está presente institucionalmente na saúde, hoje, tem se apresentado insuficiente para obter a integralidade no cuidado em saúde. Os conflitos enfrentados pelo setor saúde mostram que o trabalho nesta área requer conhecimentos mais ampliados do ser humano, que são encontrados no contexto sociocultural no qual está inserido.

Azize (2002) em sua dissertação chama a atenção para um fato importante: a biomedicina oficial é amplamente reconhecida e legitimada como um saber eficaz, que repre- senta um discurso competente de verdade, com a doença vista como naturalizada e unívoca, tomando-a como um dado da realidade, naturalizando-se a patologia na maneira como ela é definida a partir da perspectiva biomédica.

Esta compreensão gera importantes implicações ao sistema de saúde como enfatiza Ribeiro (2011) em sua dissertação de mestrado ao pontuar que a incorporação da noção ampliada de saúde que se opõe aos limites da visão biomédica restrita reconhece a necessidade de um compromisso com a implantação de serviços de saúde que privilegiem estratégias de atendimento orientadas em aspectos sociais, humanistas, culturais e ambientais.

Morais (2014) em sua tese e doutorado afirma que o estudo dos conceitos de saúde/doença junto a indivíduos e grupos específicos permite um entendimento simultaneamente específico e abrangente sobre os sentidos e significados que representam tais conceito bem como do cuidado, possibilitando adequar os serviços de prevenção e intervenção as populações e enfermidades específicas. A autora destaca também que a transição do modelo biomédico, hegemônico por muito tempo, para o biopsicossocial ainda está se efetivando, embora existam dificuldades neste processo.

\section{Perspectiva social}

A perspectiva social dos conceitos saúde e doença é ressaltada por diferentes estudos Rodrigues (2015), Carotta (1996), Azize (2002), Menezes (2010), Ferraro (2011), Dall'Olio (2006), Marchesini (2010), Maftum (2004), Osinaga (2004), Carvalho (1993), Gomes (2014), Ribeiro (2011), Grigoletto (2007), Pumar-Cantini (2005), Martins (2008), Nicareta (2010), Morais (2014) e Duarte (2014), ou seja, todas as produções acadêmicas analisadas discorrem sobre a perspectiva social de tais conceitos.

Ao discutir os resultados de sua dissertação de mestrado Ferraro (2011) argumenta que a maioria das pessoas entrevistadas em sua pesquisa possui um conceito limitado de saúde a questões meramente físicas e não consideram as dimensões social e subjetiva deste conceito. Embora a autora enfatize em seu estudo que os conceitos de saúde e doença são intrinsicamente relacionados, refletindo a conjuntura social, econômica, política e cultural de um local e época específicos.

Nessa mesma direção Andrade (1993), em sua dissertação de mestrado, enfatiza que um corpo saudável ou doente apenas é definido no conjunto das relações sociais. De acordo com a citada autora, tal reconhecimento também serviu como uma das justificativas também para intensificar 
a intervenção do Estado na vida social.

Grigoletto (2007), em sua dissertação de mestrado corrobora esta visão ao afirmar que à medida que os conceitos de saúde e doença modificam-se ao longo do tempo também são transformadas as práticas de saúde, com a incorporação do aspecto social na concepção do processo saúde-doença acarretando a necessidade de uma atuação maior do Estado sobre os determinantes sociais da saúde.

Os denominados determinantes sociais da saúde consistem nos aspectos que condicionam a saúde de uma determinada população. O estado de saúde da população é expresso predominantemente pelos dados que descrevem a incidência de doenças, lesões ou doenças. Os fatores que determinam um problema de saúde são complexos devido à natureza da correlação multifatorial, excluindo-se influências hereditárias, os determinantes sociais da saúde podem ser uma causa direta ou indireta de doença, lesão, dor ou morte (STAWARZ et al, 2014).

Ainda de acordo com Stawarz et al. (2014), vários grupos de condições sociais, econômicas e ambientais têm um impacto significativo na saúde de populações e indivíduos são referidos como determinantes indiretos que afetam a saúde em longo prazo. Em contraste, aspectos como dieta e tipo de alimentos, a qualidade da água potável, tabagismo, abuso de álcool são considerados determinantes diretos .

Nesse mesmo viés, Pumar-Cantini (2005) ressalta em sua dissertação de mestrado as múltiplas facetas que o patológico - a doença - pode assumir. Pode-se inferir, assim, que a doença assume aspectos particulares impossíveis de serem apreendidos somente por meio da teoria, uma vez que se manifesta dentro de um dado contexto individual e social, portanto para que seja compreendida, deve ser considerada inserida nestes contextos.

\section{Perspectiva subjetiva}

Diferentes autores como Rodrigues (2015), Carotta (1996), Azize (2002), Menezes (2010), Ferraro (2011), Dall'Olio (2006), Marchesini (2010), Maftum (2004), Osinaga (2004), Carvalho (1993), Gomes (2014), Ribeiro (2011), Grigoletto (2007), Pumar-Cantini (2005), Martins (2008), Nicareta (2010), Morais (2014) e Duarte (2014) examinam a perspectiva subjetiva dos conceitos saúde e doença, isto é, todas as produções acadêmicas analisadas abordam sobre a perspectiva subjetiva destes conceitos.

Rodrigues (2015) em sua tese de doutorado afirma que incorporar a subjetividade para o entendimento dos conceitos de saúde e doença implica relacionar ao corpo orgânico aos estados de prazer e dor pelos quais o ser vivo passa. Isto significa também descartar a ideia do corpo como um objeto fragmentado congruente à lógica do mecanicismo, e apreendê-lo como um todo, conforme a teoria holística, o que possibilita uma compreensão mais abrangente das relações entre a parte e o todo, bem como dos movimentos vitais. Dessa forma, o sentido existencial que a doença possui para o humano indica que a doença se dirige ao organismo por completo.

Em sua dissertação de mestrado, Dall'Olio (2006) ressalta que quando se considera a subjetividade na compreensão do processo do adoecimento, observa-se que as concepções sobre saúde e doença não podem ser entendidos de maneira linear e normatizados, mas, ao contrário precisam ser considerados no campo subjetivo, já que cada sujeito sofre sua lesão e resiste a ela de forma muito singular.

O estado patológico é sempre definido por meio da experiência individual e subjetiva e não pode ser estipulado por nenhum parâmetro exclusivamente externo. Desse modo, compreender o estado patológico requer diálogo constante com aquele que vivencia a experiência de sofrimento. Simultaneamente a concepção positiva sobre a saúde, com atenção para a capacidade normatividade do vivente - manifestada também por meio de maneiras de mobilização voltadas a valores pertinentes à dignidade da vida - possibilita muitas opções de enfrentamento a patologias (BRITO, 2017).

Nesse mesmo sentido, Grigoletto (2007) em sua dissertação de mestrado enfatiza a necessidade de se considerar a subjetividade das pessoas frente às situações vivenciadas, bem como suas dificuldades no enfrentamento de seus problemas de saúde. Pumar-Cantini (2005), em sua dissertação de mestrado, pontua também que o componente simbólico, crer em um poder de cura de algo, a subjetividade singular de cada pessoa, os variados significados construídos para as enfermidades suscitam uma necessidade afetiva e relacional que ajuda e condiciona o cuidado terapêutico próprio para cada paciente.

A identificação de uma doença depende da experiência subjetiva do mal-estar. Contudo, a partir de uma teoria positiva da saúde o conceito médico primário não é o negativo, mas o positivo, a saúde. Assim, pode ser possível descobrir a doença sem uma visão implícita sobre o que é a saúde, o objetivo de se priorizar logicamente a saúde é proveniente da consideração dos sujeitos como agentes que interagem entre si em um contexto social orientados por seus interesses. Além disso, compreende que uma avaliação da doença como incapacidade pressupõe a noção de capacidade, lógica e empiricamente anterior à primeira (GAUDENZI, 2016).

Por fim deve-se destacar, conforme Ribeiro (2011), em sua dissertação de mestrado, que a complexidade e amplitude dos conceitos de saúde e doença significa superar a hipótese de que estar saudável é não estar doente. Essa visão difundida pela Organização Mundial de Saúde de saúde como completo bem-estar biopsicossocial e não apenas ausência de enfermidade foi alvo de muitas críticas pelo caráter utópico que atribui à saúde, mas também foi útil por reconhecer a ideia da dinamicidade e complexidade do corpo humano, pois, em sua base está a compreensão de que a vida implica uma polaridade dinâmica entre saúde e doença.

\section{Conclusão}

O presente estudo permitiu analisar examinar as dissertações e teses, presentes na BDTD que possuíam como objeto de estudo os conceitos de saúde e doença.

Há limitações intrínsecas a este tipo de e revisão bibliográfica como a análise de dados secundários, ou seja, que foram encontrados por outro autor e a insuficiência de ineditismo, uma vez que discute achados de outros estudos. Contudo, como se optou por se analisar as dissertações e teses que, muitas vezes, ainda não foram publicados a limitação também pode ser vista como uma potencialidade, pois se propicia visibilidade a tais estudos acadêmicos.

Por meio da análise das doze dissertações e seis teses examinadas foram identificados três grandes temas: 
perspectiva biomédica, perspectiva social e perspectiva subjetiva. Importante destacar que tais temas não se excluem simultaneamente, mas funcionam como direcionadores para subsidiar a análise dos dados.

Embora todas as produções analisadas abordem os três temas, isto não significa que eles possuam o mesmo grau de destaque nos estudos. A perspectiva biomédica que considera somente aspectos físicos do processo saúde-doença foi identificada como a mais consolidada e hegemônica por muito tempo nas práticas de saúde.

Há o reconhecimento da relevância das perspectivas social e subjetiva para o entendimento ampliado e necessário dos conceitos de saúde e doença, no entanto, os estudos analisados também apontam para os desafios na transição de um modelo biomédico de compreensão da interface saúde e doença para um modelo abrangente de práticas em saúde que contemple as diferentes dimensões dos referidos conceitos, quer sejam, biológicas, sociais e subjetivas.

A complexidade do processo saúde/doença também foi destacada em algumas produções acadêmicas. Foi observado que a consideração de tal complexidade é fundamental para a adoção de um cuidado em saúde mais apropriado às necessidades de grupos sociais e indivíduos, uma vez que somente abordagens múltiplas e abrangentes são capazes de responder à dinamicidade inerente entre saúde e doença. Incorporar esta complexidade, contudo, representa um desafio considerável, já que até mesmo a formação dos profissionais de saúde ainda possui grande influência da perspectiva biomédica, que privilegia os aspectos físicos/objetivos do processo saúde-doença, possuindo entendimento e consequentes estratégias de ação restritos e insuficientes para as práticas em saúde.

Por fim, deve ser testacado que as posições expressas nesse artigo pertencem exclusivamente à autora e não refletem, necessariamente, a visão da instituição à qual está vinculada.

\section{Referências}

AZIZE, R. L. A química da qualidade de vida: um olhar antropológico sobre uso de medicamentos e saúde em classes médias urbanas brasileiras. Florianópolis, 2002. Dissertação (mestrado) Antropologia Social - Departamento de Antropologia, Universidade Federal de Santa Catarina.

BARDIN, L. Análise de conteúdo. Lisboa: Edições 70, 2011.

BRASIL, BDTD - BIBLIOTECA BRASILEIRA DE TESES E DISSERTAÇÕES. O que é? Disponível em: <http://bdtd. ibict.br/vufind/Content/whatIs>. Acesso em: 24 nov. 2017.

BRITO, J. Saúde - uma relação com o meio e os modos de vida. Laboreal, Porto, v. 13, n. 1, p. 100-103, jul. 2017.

CANESQUI, A. M. Reflexões sobre os conceitos de saúde e doença e suas implicações Instruções aos Colaboradores. Physis, Rio de Janeiro, v. 26, n. 1, p. 369-372, mar. 2016.

CARVALHO, Y. M. O mito atividade física/saúde. Campinas, 1993, 154 f. Dissertação (mestrado) Educação
Física - Faculdade de Educação Física, Universidade Estadual de Campinas.

CAROTTA, F. Fonoaudiologia e saúde mental: considerações sobre o trabalho fonoaudiológico em um centro de atenção psicossocial. São Paulo, 1996. Dissertação (mestrado) Fonoaudiologia - Departamento de Fonoaudiologia, Pontifícia Universidade Católica de São Paulo.

DALL'OLIO, A. M. M. O corpo adoecido em seu seio: subjetividade e câncer de mama. Fortaleza, 2006. Dissertação (mestrado) Psicologia - Centro de Ciências Humanas - CCH, Universidade de Fortaleza - UNIFOR.

DUARTE, T. C. Representações de saúde e doença por profissionais da Saúde no contexto pluricultural de fronteira. Foz do Iguaçu, 2014. 113 f. Dissertação (mestrado) Sociedade, Cultura e Fronteiras - Centro de Educação e Artes, Universidade Estadual do Oeste do Paraná.

FERRARO, M. R. M. A concepção de professores sobre saúde na escola, Ribeirão Preto, 2011. 124p. Dissertação (mestrado) Saúde na Comunidade - Faculdade de Medicina de Ribeirão Preto, Universidade de São Paulo.

GAUDENZI, P. Normal e Patológico no naturalismo e no normativismo em saúde: a controvérsia entre Boorse e Nordenfelt. Physis, Rio de Janeiro, v. 26, n. 3, p. 747-767, set. 2016.

GOMES, J. C. Atenção à saúde de gestantes, puérperas e bebês: o agir em competência de equipes de saúde na atenção primária à saúde. Porto Alegre, 2014. 112 f. Dissertação (mestrado) Ensino na Saúde - Faculdade de Medicina, Universidade Federal do Rio Grande do Sul.

GRIGOletto, J. C. Percepção de risco em relaçao à saúde bucal de indivíduos cadastrados em um Núcleo de Saúde da Família de Ribeirão Preto-SP, Ribeirão Preto, 2007. 197 p. Dissertação (mestrado) Escola de Enfermagem, Universidade de São Paulo.

MAFTUM, M. A. O ensino de enfermagem em saúde mental e psiquiátrica no Paraná. Ribeirão Preto, 2004. Tese (doutorado) Enfermagem - Escola de Enfermagem, Universidade de São Paulo.

MARCHESINI, A. L. S. A constituição do si-mesmo: uma abordagem winnicottiana. São Paulo, 2010. 85 f. Dissertação (mestrado) Psicologia Clínica - Pontifícia Universidade Católica de São Paulo.

MARTINS, A. S. R. Perfil social das pessoas com hipertensão arterial sistêmica atendidas no Programa de Saúde da Família. Franca, 2008. 123 f. Dissertação (mestrado) Serviço Social - Faculdade de História, Direito e Serviço Social, Universidade Estadual Paulista.

MENEZES, M. A Criança e sua rede familiar: significações do processo de hospitalização. Florianópolis, 2010. 411 p. 
Tese (doutorado) Psicologia - Centro de Filosofia e Ciências

MORAIS, C. A. Concepções de saúde e doença mental: adaptação transcultural do instrumento de acesso aos cuidados em saúde mental. Brasília, 2014. 140f. Tese (doutorado) Psicologia Clínica e Cultura - Departamento de Psicologia Clínica, Universidade de Brasília.

MURPHY, D. Concepts of disease and health. Revisado em 2015. Disponível em: < https://plato.stanford.edu/entries/ health-disease/>. Acesso em: 24 nov. 2017.

NICARETA, J. R. Novo BAROS: análise crítica da metodologia e sugestões para aprimoramento do BAROS. Curitiba, 2010. 230f. Tese (doutorado) Clínica Cirúrgica - Setor de Ciências da Saúde da Universidade Federal do Paraná.

NORDENFELT, L. The concepts of health and illness revisited. Medicine, Health Care and Philosophy, v. 10, n. 1, p. 05-10, mar. 2007.

OSINAGA, V. L. Estudo comparativo entre os conceitos de saúde e de doença mental e a assistência psiquiátrica, segundo portadores e familiares. Ribeirão Preto, 2004. 164 p. Tese (doutorado) Enfermagem Psiquiátrica - Escola de Enfermagem, Universidade de São Paulo.

PUMAR-CANTINI, L. Prática Curativa: Um Saber Sonegado? Rio de Janeiro, 2005. 119 p.

Dissertação (mestrado) História das Ciências e da Saúde Casa de Oswaldo Cruz, Fundação Oswaldo Cruz.

RIBEIRO, M. P. A avaliação geriátrica ampla: a contribuição da enfermagem na promoção da saúde do idoso. 2011. 117f. Dissertação (mestrado) Gerontologia - Pontifícia Universidade Católica de São Paulo, São Paulo.

RODRIGUES, J. M.; PEIXOTO JUNIOR, C. A. Reflexões sobre conceitos afirmativos de saúde e doença nas teorias de Georges Canguilhem e Donald Winnicott. Physis, Rio de Janeiro , v. 24, n. 1, p. 291-310, mar. 2014 .

RODRIGUES, J. M. Conceitos afirmativos de saúde e doença: um enlace entre os pensamentos de Georges Canguilhem e Donald Winnicott. Rio de Janeiro, 2015. 177f. Tese (doutorado) Psicologia Clínica - Departamento de Psicologia, Pontifícia Universidade Católica do Rio de Janeiro.

ROTHER, E. T. Revisão sistemática $\mathrm{x}$ revisão narrativa. Acta Paulista de Enfermagem, São Paulo, v. 20, n. 2, p. v-vi, jun. 2007.

STAWARZ, B. et al. Health and determinants of health - a review of literature. Journal of Public Health, Nursing and Medical Rescue, n. 2, p. 04-10, 2014. 